\title{
Aggregation Behaviour as a Source of Collective Decision in a Group of Cockroach-Like-Robots ${ }^{\star}$
}

\author{
Simon Garnier ${ }^{1}$, Christian Jost ${ }^{1}$, Raphaël Jeanson ${ }^{1}$, Jacques Gautrais ${ }^{1}$, \\ Masoud Asadpour ${ }^{2}$, Gilles Caprari ${ }^{2}$, and Guy Theraulaz ${ }^{1}$ \\ 1 Centre de Recherches sur la Cognition Animale, UMR-CNRS 5169, \\ Université Paul Sabatier, 118 route de Narbonne, F-31062 Toulouse cedex 4, France \\ simon.garnier@cict.fr \\ 2 Autonomous Systems Lab, Swiss Federal Institute of Technology, \\ (EPFL), CH-1015 Lausanne, Switzerland
}

\begin{abstract}
In group-living animals, aggregation favours interactions and information exchanges between individuals, and thus allows the emergence of complex collective behaviors. In previous works, a model of a self-enhanced aggregation was deduced from experiments with the cockroach Blattella germanica. In the present work, this model was implemented in micro-robots Alice and successfully reproduced the agregation dynamics observed in a group of cockroaches. We showed that this aggregation process, based on a small set of simple behavioral rules of interaction, can be used by the group of robots to select collectively an aggregation site among two identical or different shelters. Moreover, we showed that the aggregation mechanism allows the robots as a group to "estimate" the size of each shelter during the collective decision-making process, a capacity which is not explicitly coded at the individual level.
\end{abstract}

\section{Introduction}

Since the last 15 years, collective robotics has undergone a considerable development [18. In order to control the behavior of a group of robots, collective robotics was often inspired by the collective abilities demonstrated by social insects 315. Indeed, nature has already developed many strategies that solve collective problems through the decentralized organisation and coordination of many autonomous agents by self-organized mechanisms [4].

Among all these self-organized behaviours, aggregation is one of the simplest. But it is also one of the most useful. Indeed, aggregation is a step towards much more complex collective behaviours because it favours interactions and information exchanges among individuals, leading to the emergence of complex

\footnotetext{
* This work was partly supported by a European community grant given to the LEURRE project under the IST Programme (2002-2005), contract FET-OPEN-IST2001-35506 of the Future and Emerging Technologies arm and by the Programme Cognitique from the French Ministry of Scientific Research. The authors would like to thank Jean-Louis Deneubourg for all its very helpful advices about this work.
} 
and functional self-organized collective behaviours (for some examples, see [4]). As such it plays a keyrole in the evolution of cooperation in animal societies 6.

Such self-organized aggregation processes were regularly used in collective robotics. For instance, foraging tasks (i.e. clustering of objects scattered in the environment) were used to study the impact of the group size [12] or of a simple form of communication [17] on the harvest efficiency. But even more complex consequences of aggregation processes were studied with groups of robots. For instance, [1] showed that division of labor can emerge in a group of foraging robots when the size of the group grows. 8] showed that an object clustering paradigm based on stigmergy [7] can lead a group of robots to order and assemble objects of two different types.

In this paper we address a new collective behavior that is based on selforganized aggregation of robots themselves. We show that a self-enhanced aggregation process, which leads groups of cockroaches to a quick and strong aggregation [10, can be used by a group of mini-robots Alice to select collectively an aggregation site among two identical or different shelters. We show that, even though these robots have limited sensory and cognitive abilities, they are still able to perform a collective decision. It has already been shown that such self-enhanced mechanisms are used by insects to make collective decisions: for instance in food source selection in bees [16 or in resting site selection in cockroaches [2]. These collective choices appear through the amplification of small fluctuations in the use of two (or more) targets.

We first describe the biological model of aggregation we have used and the way this model was implemented in a group of mini-robots Alice. We then show that this implementation indeed results in a collective aggregation behavior that is quantitatively indistinguishable from cockroach aggregation. Finally, we show that, when this aggregation behavior is restricted to certain zones in the environment (for instance by natural preferences for dark places as in cockroaches [14]), the robots preferentially aggregate in only one of these zones, i.e. they collectively choose a single "rest" site. When these zones are of different sizes, the robots preferentially choose the biggest of the two, but without being individually able to measure their size. The results of our experiments were also used to calibrate a computer simulation model of Alice robots that will allow us to extend the exploration of this collective decision model in further studies.

\section{Self-organized Aggregation}

The aggregation process cited above is directly inspired by a biological model of displacement and aggregation developed from experiments with first instar larvae of the german cockroach Blattella germanica 910. This model was built by quantifying individual behaviors of cockroaches, that is their displacement, the interactions among individuals and with the environment in a homogeneous circular arena (11 cm diameter). Each of these individual behaviors was described in a probabilistic way: we measured experimentally the probability rate for a given behavior to happen. 
This analysis showed that cockroaches display a correlated random walk (constant rate to change direction and forward oriented distribution of turning angles) in the center of the arena [9. When reaching the periphery of the arena, cockroaches display a wall following behavior (thigmotactic behavior) with a constant rate to leave the edge and return into the central part of the arena 9]. In addition, cockroaches can stop moving at any moment, stay motionless for some time and then move again. Analysis showed that the stopping rate for an individual increases with the number of stopped cockroaches in the direct neighbourhood (within the range of antenna contact) [10]. On the other side, the rate to leave an aggregate decreases with this number [10]. Thus, this dual positive feedback leads to the quick and strong formation of aggregates (see Fig. 1). A more detailed description of the model can be found in [910].

The first part of our work was to implement this biological model of aggregation in the micro-robots Alice. These robots were designed at the EPFL (Lausanne, Switzerland) [5]. They are very small robots $(22 \mathrm{~mm} \times 21 \mathrm{~mm} \times 20 \mathrm{~mm})$ equipped with two watch motors with wheels and tires allowing a maximum speed of $40 \mathrm{~mm} \mathrm{~s}^{-1}$. Four infra-red sensors are used for obstacle detection and local communication among Alices (up to $4 \mathrm{~cm}$ distance). Robots have a microcontroller PIC16LF877 with 8K Flash EEPROM memory, 368 bytes RAM but no built-in float operations. To determine the number of neighbors (upon which the aggregation process relies), each robot owns a specific identification number and counts the number of nearby neighbors in a distance roughly less than 4 $\mathrm{cm}$. Intrinsic differences between the perception area of robots and cockroaches and imperfect neighbor counts due to noise in IR devices required some finetuning of the behavioral parameters in order for the behavioural output of the robots to correctly match the cockroach individual behaviors. This behavioral output of robots was measured using the same experimental methods (10 to 30 experiments depending on the studied behavior) as those used to characterize the individual behavior of cockroaches 910 .

However the true validation of the model implementation must be done at the collective level by comparing the aggregation behavior of robots to the aggregation behavior of cockroaches. To this aim, we ran the following aggregation experiment: groups of robots (10 or 20 individuals) were put into a homogeneous white circular arena (50 cm diameter) during 60 minutes. This experiment is similar to the one done by [10] with cockroaches. To draw a parallel between cockroach aggregation behavior and robot aggregation behavior, we scaled the dimensions of the arena so that it matches scale differences between robot and cockroach sizes. The experiment was repeated 10 times for each group size. The aggregation dynamics were characterized through three kinds of measurements (sampled every minute): size of the largest aggregate, number of aggregates and number of isolated individuals (see 910] for a detailed description of these measurements). The experimental results showed a very good agreement between robots and cockroaches, confirming that the cockroach aggregation process was well implemented in the Alice robots (see Fig. 1). 


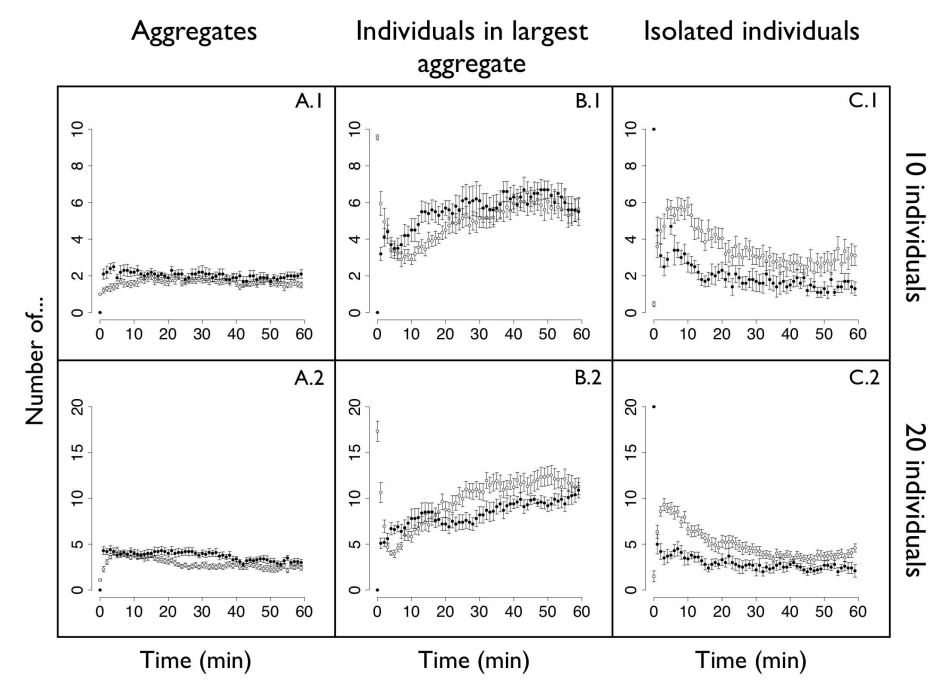

Fig. 1. Aggregation dynamics.A: number of aggregates. B: size of the largest aggregate. C: number of isolated individuals. 1: experiments with 10 individuals. 2: experiments with 20 individuals. Black dots represent data for robots; white dots represent data for cockroaches. Each dot represents the mean \pm standard error (s.e.). Initial differences between starting points of robot and cockroach dynamics are solely due to the way cockroaches have to be brought into the arena as explained in 10.

\section{Collective Choice}

This aggregation process implemented in robots can occur anywhere in the whole experimental arena, with no preference for a given location. Actually, in nature some places are more attractive for cockroaches, thus promoting aggregation in particular sites. For instance, cockroaches preferentially aggregate in dark places 14. Experimentally, if one puts a dark shelter in a lighted arena (as the one used for the study of cockroach aggregation), one can observe that cockroaches strongly aggregate under this shelter. And if two or more dark shelters are placed in the arena, one can observe that a majority of cockroaches aggregates under only one of these shelters, rather than spreading evenly among all the aggregation sites [1]. Hence cockroaches are able to perform a collective choice for a given aggregation site, even if these sites are identical.

Though the mechanisms leading to this collective choice are not yet fully understood, we suggest that this choice could strongly rely on the self-enhanced aggregation process described above and tested with robots. In a recent paper, [2] showed that the simple modulation of the resting period on a given site by the number of individuals on that site leads the group of cockroaches to the choice of one shelter among two or more identical ones. We suggest here that this modulation can be implemented easily through the aggregation process described above. To test our hypothesis, we ran three sets of experiments during which a 


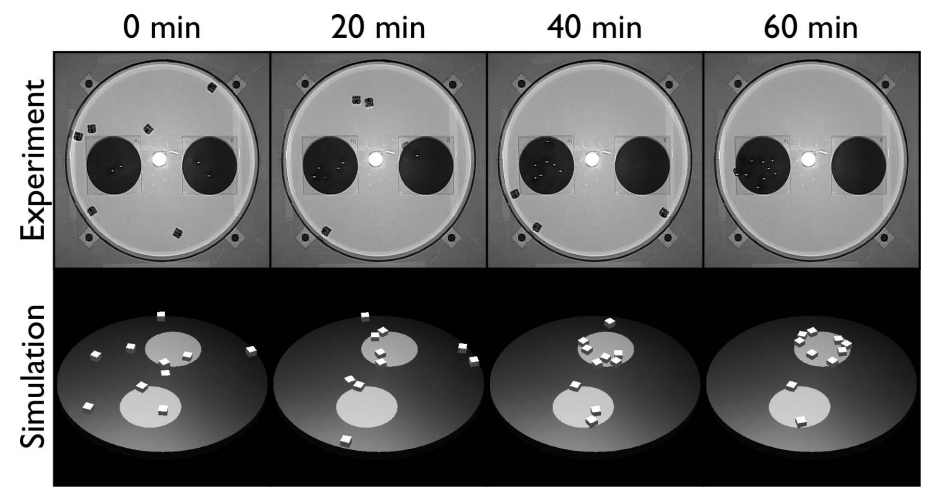

Fig. 2. Snapshots of an experiment (top) and a simulation (bottom) taken every 20 minutes during 60 minutes. These snapshots correspond to the experiment with two identical shelters (14 cm diameter). As can be seen, the experiment ended with the choice of one of the two shelters by both real and simulated robots.

group of robots faced the choice between two potential aggregation sites. Besides proving that a collective decision can appear in robots from a simple aggregation process, these experiments were also used to calibrate a simulation tool which will be used in further studies to identify the behavioral parameters that control collective choice (see Fig. 2 for some pictures of both experiments wih robots and simulations). In the following, all statistical computations will be made in the free software $\mathrm{R}[13$.

The first set of experiments was designed to ascertain whether the cockroach aggregation behavior is able to lead a group of robots to a collective choice between two identical targets. To that aim, we put a group of 10 robots in the same arena as the one used for aggregation experiments, except that we added just above the arena two dark shelters. These shelters were of the same size (14 cm diameter) and each of them can house the whole population of robots. Robots used the same behavioral algorithm as the one previously tested for its aggregation ability, except that, now, robots only stop under dark shelters (that is when IR light intensity falls under a given threshold). 20 experiments were performed, each lasting 60 minutes.

The number of stopped robots under each shelter was measured every minute to characterize the aggregation dynamics under each shelter. In addition, we also computed the percentage of stopped robots under each shelter at the end of each experiment to characterize the collective choice of the group of robots. From this last measurement, we derive what we call a "choice distribution". For a given shelter, this choice distribution corresponds to the number of experiments ending with a given percentage of stopped robots under this shelter (the choice distribution being symmetrical for the other shelter). Note that a robot can be in one of these three locations at the end of an experiment: under shelter 1, under shelter 2 or outside the shelters. In the case of each robot choosing randomly a shelter (i.e. without any influence of its conspecifics), the result will follow a trinomial law 
with parameters $m_{t o t}=10$ (number of robots), $p_{a}=\left(m_{t o t}-m_{s}\right) / m t o t\left(p_{a}\right.$, probability for a robot to be outside the shelters; $m_{s}$ number of robots stopped under any shelter, estimated from the experiments), $p_{s 1}=\left(1-p_{a}\right)\left(r_{s 1}^{2} /\left(r_{s 1}^{2}+r_{s 2}^{2}\right)\right)\left(p_{s 1}\right.$, probability for a robot to be under shelter $1 ; r_{s 1}$, radius of shelter $1 ; r_{s 2}$, radius of shelter 2) and $p_{s 2}=1-p_{s 1}-p_{a}\left(p_{s 2}\right.$, probability for a robot to be under shelter 2). The choice distribution resulting from this trinomial law can be obtained through Monte Carlo simulations (10000 simulations of 20 replicates). In the case of identical shelters, this choice distribution displays a centered peak as can be seen in Fig. 3 B.1, meaning that a majority of experiments should end with no choice for a particular shelter.

Contrary to the trinomial resulting choice distribution, the choice distribution obtained in experiments with two identical shelters displays two peaks, one at each side (see Fig. 3 B.2). A chi square test shows a strong difference between the trinomial and experimental distributions $\left(\chi^{2}=367.7, d f=4, p<0.0001\right)$. Similar results are obtained with simulations (see Fig. 3 B.3) and a chi square analysis of contingency tables shows no difference between experiments and simulations $\left(\chi^{2}=2.1, p=0.7322\right.$, p-value simulated with 10000 replicates $)$. This U-shape distribution corresponds to two different "populations of experiments", each of them preferentially ending with the choice of one of the two shelters. Furthermore, in this case with two identical shelters, the symmetry of the U-shape means that each shelter is randomly chosen from one experiment to another. The dynamics of this choice can be seen in Figs. 4 B.1 and B.2. It shows that the choice occurs very rapidly within the first minutes of the experiments. It also shows that this choice is very strong, since $75.5 \pm 3.36 \%$ (mean \pm s.e., $n=20$ ) of the population of robots is under the chosen shelter at the end of the experiments $(78 \pm 0.53 \%, n=1000$, in simulations). Thus this set of experiments clearly shows that the aggregation process described above (with very simple individual behaviors) can lead a group of robots to perform a collective choice between two aggregation sites.

The two other sets of experiments were designed to assess the impact of a qualitative difference between the two shelters on the collective choice. As in the previous set of experiments, a group of 10 robots faced a choice between two shelters. But this time, while one of the shelters kept the same size as in the previous experiment, the size of the other was altered.

In a first set of 20 experiments, we confronted a $14 \mathrm{~cm}$ diameter shelter (able to house the whole robot population) with a $10 \mathrm{~cm}$ diameter shelter (too small to house the whole population of robots). As can be seen in Figs. 4 A.1 and A.2, robots quickly and strongly choose the shelter able to house their whole population. Thus, at the end of the experiments, $68 \pm 3.29 \%$ (mean \pm s.e., $n=20)$ of the population is under the $14 \mathrm{~cm}$ diameter shelter $(72.7 \pm 0.79 \%$, $n=1000$, in simulations). The choice distribution shows a strong shift towards the $14 \mathrm{~cm}$ diameter shelter (see Fig. 3 A.2). This shift is the result of more than the simple difference between the area of the two shelters. Indeed, a comparison between the experimental distribution and a trinomial distribution (Fig. 3 A.1) taking into account this difference in size shows a strong difference $\left(\chi^{2}=365.4\right.$, 


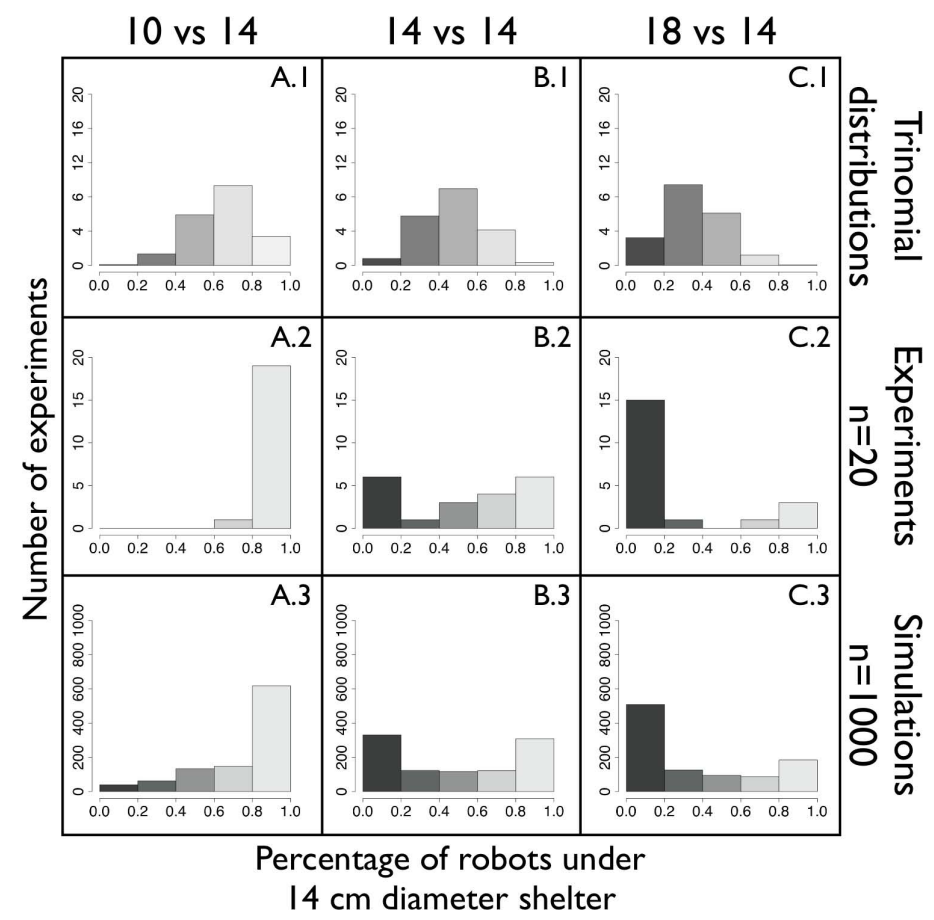

Fig. 3. Choice distributions. In these distributions, each block represents a number of experiments ending with a given percentage (0-20, 20-40, 40-60, 60-80 and 80-100 percent) of robots under one of the two shelters. Top: trinomial distributions (random choice). Middle: experimental distributions $(n=20)$. Bottom: simulation distributions $(n=1000)$. Columns $\mathbf{A}$ and $\mathbf{C}$ represent choice distributions for the $14 \mathrm{~cm}$ diameter shelter against either the $10 \mathrm{~cm}$ diameter shelter (column A) or the $18 \mathrm{~cm}$ diameter shelter (column C). For each of these distributions, blocks on the right mean choice of the $14 \mathrm{~cm}$ diameter shelter and blocks on the left mean choice of the other shelter (either 10 or $18 \mathrm{~cm}$ diameter). Column B represents the choice distribution for a $14 \mathrm{~cm}$ diameter shelter against an other $14 \mathrm{~cm}$ shelter.

$d f=4, p<0.0001$ ). Similar results are obtained with simulations (see Fig. 3 A.3) and a chi square analysis of contingency tables shows no difference between experiments and simulations $\left(\chi^{2}=9.4, p=0.0595\right.$, $\mathrm{p}$-value simulated with 10000 replicates [13]). The disappearance of the U-shape of the distribution means that it remains only one "population of experiments" preferentially ending with the choice of the $14 \mathrm{~cm}$ diameter shelter, i.e. the one able to house the whole population of robots.

In a second set of 20 experiments, we confronted a $14 \mathrm{~cm}$ diameter shelter with a $18 \mathrm{~cm}$ diameter shelter. Both shelters are able to house the whole population of robots. As can be seen in Figs. 4 C.1 and C.2, robots choose the $18 \mathrm{~cm}$ diameter shelter. Thus, at the end of the experiments, $70.5 \pm 7.56 \%$ (mean \pm s.e., $n=20)$ of the population is under the $18 \mathrm{~cm}$ diameter shelter $(61 \pm 1.12 \%$, 


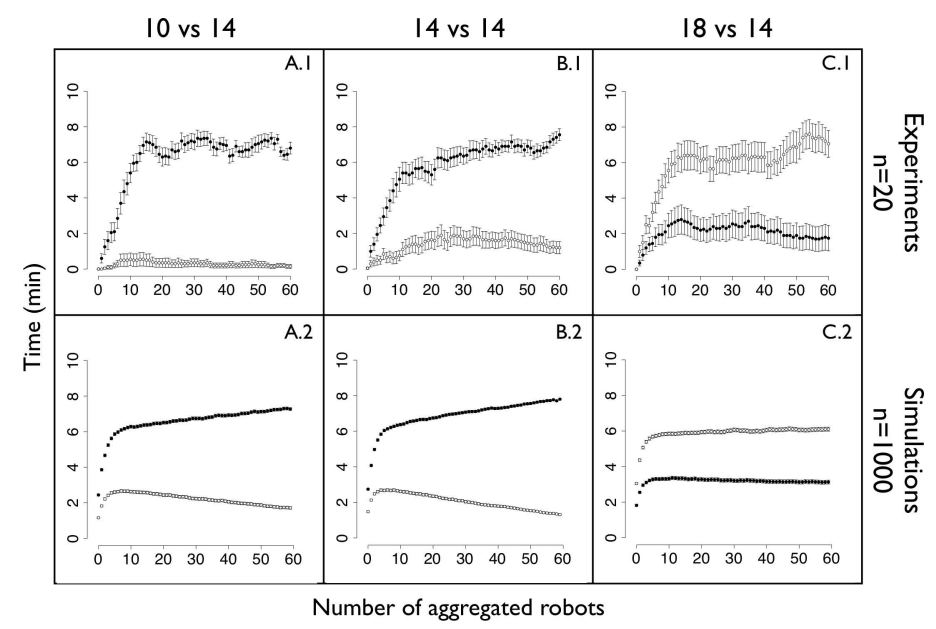

Fig. 4. Choice dynamics: number of robots aggregated under each shelter. Top: experimental data $(n=20)$. Bottom: simulation data $(n=1000)$. In column A and C, black dots represent data for the $14 \mathrm{~cm}$ diameter shelter; white dots represents data for either the $10 \mathrm{~cm}$ diameter shelter (column A) or the $18 \mathrm{~cm}$ diameter shelter (column C). In column B, black dots represent data for the chosen shelter (i.e. the shelter which is chosen at the end of each experiment); white dots represent data for the "not chosen" shelter. In all cases, each dot represents the mean \pm s.e.

$n=1000$, in simulations). The choice distribution shows a shift towards the 18 $\mathrm{cm}$ diameter shelter (see Fig. 3 C.2). This shift is the result of more than the simple difference between the area of the two shelters. Here also the comparison between the experimental distribution and a trinomial distribution (Fig. 3 C.1) taking into account this difference in size shows a strong difference $\left(\chi^{2}=373.8\right.$, $d f=4, p<0.0001$ ). And similar results are obtained with simulations (see Fig. 3 C.3) and a chi square analysis of contingency tables shows no difference between experiments and simulations $\left(\chi^{2}=5.4, p=0.2301\right.$, p-value simulated with 10000 replicates). But contrary to the previous experiment, the U-shape of the distribution has not disappeared and the two "populations of experiments" still exist: one that preferentially ended by a choice of the $14 \mathrm{~cm}$ diameter shelter, the other that preferentially ended by a choice of the $18 \mathrm{~cm}$ diameter shelter, the latter prevailing on the former.

From the two latter sets of experiments, we can conclude that the group of robots will choose preferentially a shelter that is sufficiently large to house all its members. But when the group is confronted with two sufficiently large shelters, the self-enhanced aggregation mechanism can lead the group to two stable choices, with a preference for the larger shelter. This implies that the group of robots is able to "sense" and "compare" the size of the shelters during the collective decision process, a performance that is beyond the direct scope of the simple aggregation process used in these experiments and that is not explicitly implemented in individual robots. We hypothesise that this relies on the higher 
probability for the robots to encounter this shelter in the arena. Indeed, the more robots encounter a shelter, the more likely they will stop spontaneously under it. Thus, there will be more individual stopped robots under the bigger shelter that will act as "seeds" for new clusters.

\section{Conclusion}

In this work, we achieved the implementation of a biological model of selfenhanced aggregation in a group of mini-robots Alice. Despite the strong differences in terms of sensory abilities between biological and artificial models, the aggregation dynamics observed in robots closely match those observed in cockroaches. This result is obtained by measuring robot and cockroach behaviours in terms of behavioural probabilities, thus taking into account sensory and motor abilities of the two systems. Then, by calibrating the behavioural probabilities programmed in the robots, we reproduced both individual displacement and stop behaviours of the biological system with the artificial one. And the aggregation dynamics emerge from these individual behaviours, as is expected from the model described in [9]10]. With this method, it is thus only required that the robot features approximatively reproduce cockroach features to accurately reproduce their aggregation behaviour.

Moreover, we achieved a collective decision process from this simple biological model of aggregation. We showed that a self-enhanced aggregation process associated with a preference for a given type of environmental heterogeneity (here a preference for dark places) can lead a group of robots to a collective choice for an aggregation site. Furthermore, this choice can be related to a collective ability to "sense" and "compare" the sizes of the aggregation sites. This is a very interesting robotics example of an interaction between a simple self-organized mechanism and an evironmental template, leading to the emergence of a far more complex collective behaviour and of new collective abilities not explicitly coded in the basic model of aggregation.

This work opens some interesting perspectives for collective robotics. Collective choices could be associated, for instance, with an ordering behavior of the same kind than the one described in [8], so that robots would assemble objects of different types in different places. We argue that such associations are new challenges to take up if this collective robotics, based on self-organized mechanisms and/or biologically inspired behaviors, must become an efficient and robust way to achieve complex tasks with groups of numerous small autonomous robots.

\section{References}

1. W. Agassounon and A. Martinoli. A macroscopic model of an aggregation experiment using embodied agents in groups of time-varying sizes. In Proceedings of the 2002 IEEE Systems, Man and Cybernetics Conference, Hammamet, Tunisia, 2002. IEEE Press.

2. J.-M. Ame, C. Rivault, and J.-L. Deneubourg. Cockroach aggregation based on strain odour recognition. Animal Behaviour, 68(4):793-801, 2004. 
3. E. Bonabeau, M. Dorigo, and G. Theraulaz. Swarm intelligence : from natural to artificial systems. Oxford University Press, Oxford, 1999.

4. S. Camazine, J.L. Deneubourg, N. R. Franks, J. Sneyd, G. Theraulaz, and E. Bonabeau. Self-organization in biological systems. Princeton University Press, Princeton, 2001.

5. G. Caprari, T. Estier, and R. Siegwart. Fascination of down scaling - Alice the sugar cube robot. Journal of Micromechatronics, 1(3):177-189, 2002.

6. J. L. Deneubourg, A. Lioni, and C. Detrain. Dynamics of aggregation and emergence of cooperation. Biological Bulletin, 202(3):262-7, 2002.

7. P.-P. Grassé. La reconstruction du nid et les coordinations inter-individuelles chez Bellicositermes Natalensis et Cubitermes sp. La théorie de la stigmergie : essai d'interprétation du comportement des termites constructeurs. Insectes sociaux, 6:41-81, 1959.

8. O. Holland and C. Melhuish. Stigmergy, self-organisation, and sorting in collective robotics. Artificial Life, 5:173-202, 1999.

9. R. Jeanson, S. Blanco, R. Fournier, J. L. Deneubourg, V. Fourcassié, and G. Theraulaz. A model of animal movements in a bounded space. Journal of Theoretical Biology, 225(4):443-451, 2003.

10. R. Jeanson, C. Rivault, J.-L. Deneubourg, S. Blanco, R. Fournier, C. Jost, and G. Theraulaz. Self-organized aggregation in cockroaches. Animal Behaviour, 69(1):169-180, 2005.

11. A. Ledoux. Étude experimentale du grégarisme et de l'interattraction sociale chez les Blattidés. Annales des Sciences Naturelles Zoologie et Biologie Animale, 7:76103, 1945.

12. A. Martinoli and F. Mondada. Collective and cooperative group behaviours: biologically inspired experiments in robotics. In O. Khatib and J. K. Salisbury, editors, Proceedings of the Fourth International Symposium on Experimental Robotics, pages 3-10, Stanford, June 1995. LNCIS.

13. $\mathrm{R}$ Development Core Team. $R$ : A language and environment for statistical computing. R Foundation for Statistical Computing, Vienna, Austria, 2004. ISBN 3-900051-07-0.

14. M. K. Rust, J. M. Owens, and D. A. Reierson. Understanding and controlling the german cockroach. Oxford University Press, Oxford, 1995.

15. E. Sahin. Swarm robotics: From sources of inspiration to domains of application. LNCS, 3342:10-20, 2005.

16. T.D. Seeley, S. Camazine, and J. Sneyd. Collective decision-making in honey bees: how colonies choose among nectar sources. Behavioural Ecology and Sociobiology, 28:277-290, 1991.

17. K. Sugawara and M. Sano. Cooperative acceleration of task performance: foraging behavior of interacting multi-robots system. Physica D: Nonlinear Phenomena, 100(3/4):343-354, 1997.

18. I. A. Wagner and A. M. Bruckstein. Ant robotics. Annals of Mathematics and Artificial Intelligence, 31:1-238, 2001. 